University of Nebraska - Lincoln

DigitalCommons@University of Nebraska - Lincoln

3-1-1999

\title{
Molecular dynamics study of a phase-separating fluid mixture under shear flow
}

Ryoichi Yamamoto

Kyoto University, Kyoto 606-8502, Japan

Xiao Cheng Zeng

University of Nebraska-Lincoln, xzeng1@unl.edu

Part of the Chemistry Commons

Yamamoto, Ryoichi and Zeng, Xiao Cheng, "Molecular dynamics study of a phase-separating fluid mixture under shear flow" (1999). Xiao Cheng Zeng Publications. 60.

https://digitalcommons.unl.edu/chemzeng/60

This Article is brought to you for free and open access by the Published Research - Department of Chemistry at DigitalCommons@University of Nebraska - Lincoln. It has been accepted for inclusion in Xiao Cheng Zeng Publications by an authorized administrator of DigitalCommons@University of Nebraska - Lincoln. 


\title{
Molecular dynamics study of a phase-separating fluid mixture under shear flow
}

\author{
Ryoichi Yamamoto ${ }^{1}$ and Xiao Cheng Zeng ${ }^{2}$ \\ ${ }^{1}$ Department of Physics, Kyoto University, Kyoto 606-8502, Japan \\ ${ }^{2}$ Department of Chemistry, University of Nebraska-Lincoln, Lincoln, Nebraska 68588
}

(Received 27 July 1998; revised manuscript received 14 October 1998)

\begin{abstract}
Molecular dynamics simulation is carried out to study domain structures and rheological properties of a two-dimensional phase-separating binary fluid mixture under shear flow. In the early stage of the phase separation, anisotropic composition fluctuations appear immediately after the quench. As the domain grows, the anisotropy in the composition fluctuations increases. The quenched system eventually reaches a dynamical steady state, in which anisotropic domain structures are preserved. In the steady state, the shortest characteristic length scale $R_{\perp}$ of domains decreases with increasing shear rate $\dot{\gamma}$ as $R_{\perp} \sim \dot{\gamma}^{-1 / 3}$. Stringlike domain structures are observed in the strong shear regime, whereas randomly fluctuating patterns are observed in the weak shear regime. Moreover, the excess viscosity $\Delta \eta$ is found to decrease with increasing shear rate as $\Delta \eta \sim \dot{\gamma}^{-1 / 2}$, indicating that the phase-separating fluid mixtures are highly non-Newtonian because of domain deformations.
\end{abstract} [S1063-651X(99)10703-7]

PACS number(s): 83.20.Jp, 64.75.+g, 83.50.Ax

\section{INTRODUCTION}

Spinodal decomposition occurs when a uniform system is suddenly quenched into the unstable two-phase region. Spatial inhomogeneities then emerge, grow into larger domains, and eventually arrive at a two-phase equilibrium system. In the past three decades, phase separation has been extensively studied [1], both experimentally and theoretically. A number of computer simulations [2-9] have also been carried out. These studies not only provide physical insights into the complex behavior, but also have yielded quantitative results. Among others, the most significant findings are the domain growth laws and the scaling properties of the structure factor in the spinodal decomposition. It is remarkable that mutually consistent results on the domain growth have been obtained using quite different simulation methods, including timedependent Ginzburg-Landau [2,3], lattice Boltzmann [4,5], cellular automata [6], and molecular dynamics (MD) [7-9]. A notable example is the growth law $R \sim t^{\alpha}$ for the characteristic length $R$ of the fluctuation in the late stage. It is found that the exponent $\alpha \simeq \frac{2}{3}$ and $\alpha \simeq 1$ for two-dimensional (2D) and three-dimensional (3D) fluid mixtures, respectively.

Most computer simulation studies have thus far focused on isotropic phase separation, namely, phase separation under no external field. Behaviors of phase separation under external fields such as a velocity or temperature gradient remain largely unexplored. It is expected that phase separation behaviors under external fields are qualitatively different from the universal behavior in isotropic cases [10]. For example, if a shear flow is imposed in the course of spinodal decomposition, the domain pattern will be anisotropic, that is, $R_{\|} / R_{\perp}>1$, where $R_{\|}$is the longest characteristic length scale of domains and $R_{\perp}$ is the shortest one. It is also expected that the tendency of minimizing the surface free energy (favoring growth of $R_{\perp}$ ) and the tendency of breaking up of domains by the flow (suppressing growth of $R_{\perp}$ ) can balance each other at a steady state so that $R_{\perp}$ remains finite.

Recently, the spinodal decomposition under shear has been examined in a number of experimental studies, particu- larly in domain structures and rheological properties [1113]. Analytical approaches $[14,15]$ have also been developed. However, because of the complexity of the problem, satisfactory understanding has not yet been fully achieved theoretically. On the other hand, computer simulation promises to shed some light on the spinodal decomposition under flow fields [16-19].

The purpose of this paper is to study the dependence of $R_{\perp}$ on the shear rate as well as the features of the domain structure in the steady state $\left(R_{\|}\right.$is not examined here because it can quickly reach the size of the simulation system). Another quantity considered in this paper is the excess viscosity $\Delta \eta \equiv \eta_{\text {mix }}-\eta_{\text {one }}$, where $\eta_{\text {mix }}$ is the viscosity of a fluid mixture and $\eta_{\text {one }}$ is the viscosity of the one-component fluid at equilibrium states. This quantity characterizes the domain contribution and can exhibit strong non-Newtonian character if the domains are strongly deformed by the shear. We note that Padilla and Toxvaerd have studied a similar system by MD simulation [19]. That work focused on the timedependent properties of spinodal decompositions in the presence of shear flow. Here we mainly investigate domain structures and rheological properties in the steady state.

\section{SIMULATION}

We have performed MD simulations for a 2D fluid mixture. The mixture is composed of two particle species, $A$ and $B$, which interact with each other via the Lennard-Jones (LJ) potential,

$$
\phi_{i j}=4 \epsilon\left[\left(\frac{\sigma}{r_{i j}}\right)^{12}-\delta_{i j}\left(\frac{\sigma}{r_{i j}}\right)^{6}\right]
$$

where $r_{i j}$ is the interparticle separation, $\delta_{i j}=1$ if the $i$ th and $j$ th particles are the same type, and $\delta_{i j}=0$ if those are different. For simplicity, here the two species are chosen to be identical; both have the same mass $m$, size $\sigma$, and interaction parameter $\epsilon$. All physical quantities are dimensionless in this paper. Those are reduced based on $m, \sigma$, and $\epsilon$ [20]. The potentials are truncated at $r_{i j}=2.5$. The total number of par- 
ticles $N=N_{A}+N_{B}$ is 30000 ; the number fraction $x_{A}$ $=N_{A} / N$ is taken to be 0.5 (the critical composition); the number density $\rho$ is set to 0.8 . The immiscibility between $A$ and $B$ components is introduced by removing the attractive part of interaction for unlike pairs. Although the precise value of the critical temperature $T_{c}$ of the system is unknown, $T_{c}$ was roughly estimated between 5 and 10. All simulations were carried out for the temperature $T=1$ at which $A$ and $B$ components of the fluid mixture are strongly immiscible.

For a system under flow, the temperature of the system must be monitored and controlled to eliminate the viscous heating effect. However, the particle velocity $\mathbf{v}_{i}(t)$ contains two contributions: the thermal velocity $\mathbf{v}_{i}^{t h}(t)$ and the instantaneous streaming velocity $\mathbf{u}\left(\mathbf{r}_{i}(t), t\right)$. The latter is given by

$$
\mathbf{u}\left(\mathbf{r}_{i}(t), t\right)=\frac{\sum_{j=1}^{N} \mathbf{v}_{j}(t) \delta\left(r_{i j}\right)}{\sum_{j=1}^{N} \delta\left(r_{i j}\right)},
$$

where $\delta\left(r_{i j}\right)=0$ for $r_{i j}>d$ and $\delta\left(r_{i j}\right)=1$ for $r_{i j} \leqslant d$. Here, $d=8$ is taken. We found the simulation results are insensitive to the choice of $d$ as long as $1<d \leqslant R_{\perp}$. The thermal velocity, which was actually used for calculating the temperature, is given by

$$
\mathbf{v}_{i}^{t h}(t)=\mathbf{v}_{i}(t)-\mathbf{u}\left(\mathbf{r}_{i}(t), t\right)
$$

Specifically, the simulations were carried out as follows. (i) The system was equilibrated at $T=20$ and at a steady shear rate $\dot{\gamma}=0.02-1.0$. The Lees-Edwards moving boundary condition [21] was adopted to enforce the steady shear flow. (ii) At time $t=0$, the system was quenched instantaneously to a lower temperature $T=1$ via scaling the thermal velocity $\mathbf{v}_{i}^{\text {th }}$ by a factor $\sqrt{1 / 20}$, while $\mathbf{u}\left(\mathbf{r}_{i}(0), 0\right), \dot{\gamma}$ and $\rho$ were fixed. (iii) The simulations proceeded at the fixed temperature $T=1$. The Gaussian constraint method was employed to keep the temperature [22] fixed. The leapfrog algorithm [23] was used to solve the differential equations with an integration time step $\Delta t=0.0025$ for $T=20$ or 0.005 for $T=1$. Since we do not assume any profiles of streaming velocity a priori, the thermostat is in fact the profile unbiased thermostat (PUT) [24,25]. The use of PUT is essential in the present study because the instantaneous flow field is not uniform but fluctuating in time and space due to the existence of domains. The magnitude of fluctuation is about $\left\langle\left(\delta \mathbf{u}_{i}\right)^{2}\right\rangle=0.2$ to 0.5 (depending on $\dot{\gamma}$ ), where

$$
\delta \mathbf{u}_{i}=\mathbf{u}\left(\mathbf{r}_{i}(t), t\right)-\dot{\gamma} y_{i} \mathbf{e}_{x}
$$

and $\mathbf{e}_{x}$ is a unit vector $(1,0)$. It is found that a higher shear rate leads to a larger fluctuation in $\delta \mathbf{u}_{i}$. It is also found that the eddy viscosity $\eta_{E}=\left\langle\rho u_{x} u_{y}\right\rangle / \dot{\gamma}$ accounts for nearly $10 \%$ of the total viscosity in $\eta_{\text {mix }}$, whereas $\eta_{E}$ is negligible in $\eta_{\text {one }}$.

\section{RESULTS}

We note that phase separation under shear flow can be divided into four regimes according to the magnitude of Reynolds number $\operatorname{Re}=\dot{\gamma} R_{\perp}^{2} \rho / \eta$ and the reduced shear rate $\dot{\gamma} \tau_{\xi}$ : (i) $\operatorname{Re}>1$ and $\dot{\gamma} \tau_{\xi}<1$, (ii) $\operatorname{Re}>1$ and $\dot{\gamma} \tau_{\xi}>1$, (iii) $\operatorname{Re}<1$ and $\dot{\gamma} \tau_{\xi}<1$, and (iv) $\operatorname{Re}<1$ and $\dot{\gamma} \tau_{\xi}>1$, where $\tau_{\xi}$ is the relaxation time of the composition fluctuations. The regimes $\dot{\gamma} \tau_{\xi}<1$ and $\dot{\gamma} \tau_{\xi}>1$ normally refer to the so-called weak and strong shear regimes. In the present study, $\tau_{\xi}$ is determined by the growth rate of the $I\left(k_{m}, t\right)$ in a quiescent state $[12,26]$. Here $I(k, t)$ is the scattering intensity determined by taking an angler average of the scattering function

$$
S(\mathbf{k}, t)=\frac{1}{N_{A}}\left\langle\left|\sum_{i}^{N_{A}} \exp \left(i \mathbf{k} \cdot \mathbf{r}_{i}\right)\right|^{2}\right\rangle,
$$

and $k_{m}(\simeq 0.9$ in the present case) is the peak wave number in an early stage spinodal decomposition. We found $\tau_{\xi} \simeq 8$ and $\operatorname{Re} \simeq 10$. Thus, our simulations for three low shear rates $(\dot{\gamma}$ $=0.02,0.05$, and 0.1 ) correspond to regime (i), while those for three high shear rates $(\dot{\gamma}=0.2,0.5$, and 1.0) correspond to regime (ii). Note also that phase separations studied in most experiments so far correspond to the low Reynolds number regime (iii) or (iv).

\section{A. Domain structures}

In Figs. 1-6, we show the time evolution of domain structures during phase separation for six different shear rates: $\dot{\gamma}=0.02,0.05,0.1,0.2,0.5$, and 1.0. For illustrative purpose only the distribution of $A$ particles is shown. The corresponding scattering patterns $S(\mathbf{k}, t)$ are also displayed in these figures. Note that the velocity gradient is imposed in the vertical $(y)$ direction and the flow is in the horizontal $(x)$ direction. In the early stage, it is found that the composition fluctuations appear immediately after the quench and then evolve as in usual spinodal decomposition. However, large anisotropies in composition fluctuations are also observed. The higher the shear rate, the larger the anisotropies appear. This is clearly seen in the scattering patterns at time $t$ $=12.5$ after the quench. Moreover, when the domain grows the anisotropies in the composition fluctuations increase greatly, and as a result, the scattering patterns almost collapse to the $y$ axis. To qualitatively explain this behavior we invoked a dimensional analysis (based on the Navier-Stokes equation), $\tau_{\text {relax }}=\eta R / \sigma_{s}$, where $\tau_{\text {relax }}$ is a characteristic time scale of motions in a fluid system with a length scale $R$, viscosity $\eta$, and surface (line) tension $\sigma_{s}$ [27]. It indicates that larger domains have a longer relaxation time, and thus larger domains should be deformed more strongly by the shear.

As mentioned above, $R_{\perp}$ is expected to remain finite in the steady state under shear flow, although this has not yet been fully confirmed. We attempted to verify this via the computer simulation. To this end, a large-scale $(L \simeq 200$, where $L$ is the cell size) simulation was carried out for a long time until the steady state was realized $(t \sim 1000$ at $\dot{\gamma}$ $=0.02)$. Domain structures at the end of the simulations are shown in the bottom of Figs. $1-6$. We found that $R_{\perp}$ indeed 

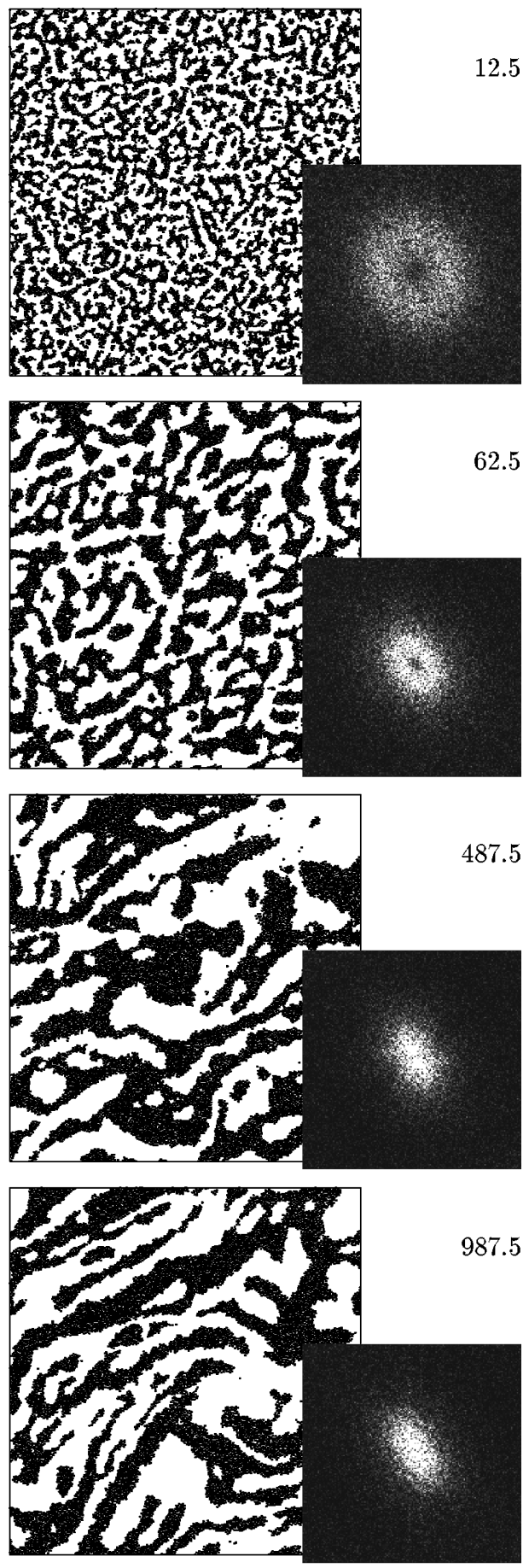

FIG. 1. Time evolution of the domain structure at $\dot{\gamma}=0.02$. The system length $L$ is 193.6. The corresponding scattering patterns $S(\mathbf{k}, t)$ are also shown with $-2.6 \leqslant k_{x}, k_{y} \leqslant 2.6$.

remains finite under all shear rates, both in the weak and strong shear regimes, and decreases as the shear rate increases. To obtain time evolutions and shear-rate dependence of $R_{\perp}$, we calculated the first moment $k_{1}(\theta)$ of the scattering patterns by fitting simulation data with the elliptic function,

$$
\left[k_{1}(\theta)\right]^{-2}=\left(\frac{R_{\perp}}{2 \pi}\right)^{2} \cos ^{2}\left(\theta-\theta_{0}\right)+\left(\frac{R_{\|}}{2 \pi}\right)^{2} \sin ^{2}\left(\theta-\theta_{0}\right),
$$

where $R_{\perp}, R_{\|}$, and $\theta_{0}$ are fitting parameters. Time evolu-
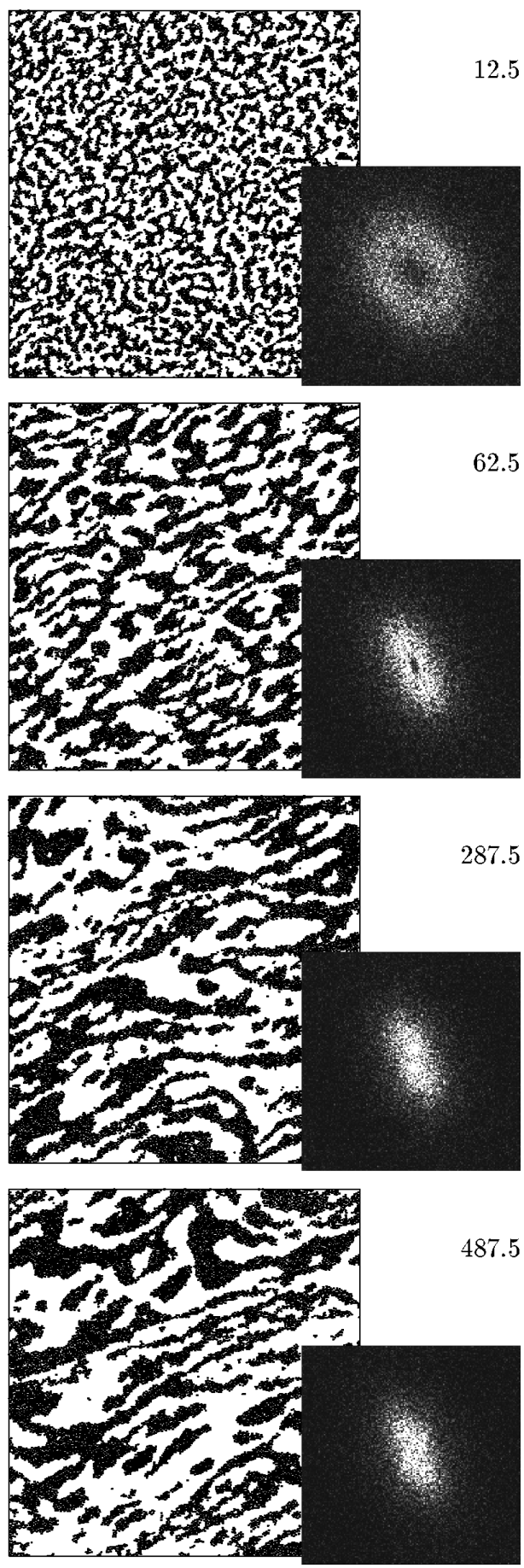

FIG. 2. Time evolution of the domain structure and the scattering patterns at $\dot{\gamma}=0.05$.

tions of $R_{\perp}$ with $\dot{\gamma}=0,0.02$, and 0.05 are shown in Fig. 7, from which one can see that the steady state is realized with shear flow. In the following parts, we analyze properties in the steady state. Data are taken by averaging many instantaneous values, and error bars indicate variances of the data. Figure 8 shows the shear rate dependence of $R_{\perp}$. Interestingly, the data support the power-law relation $R_{\perp} \sim \dot{\gamma}^{-1 / 3}$ which holds in both weak and strong shear regimes; no clear tendency of a kinetic phase transition is detected in $R_{\perp}$ around $\dot{\gamma} \tau_{\xi} \simeq 1$.

Figures 1 and 2 also show that when the shear rate $\dot{\gamma} \tau_{\xi}$ 

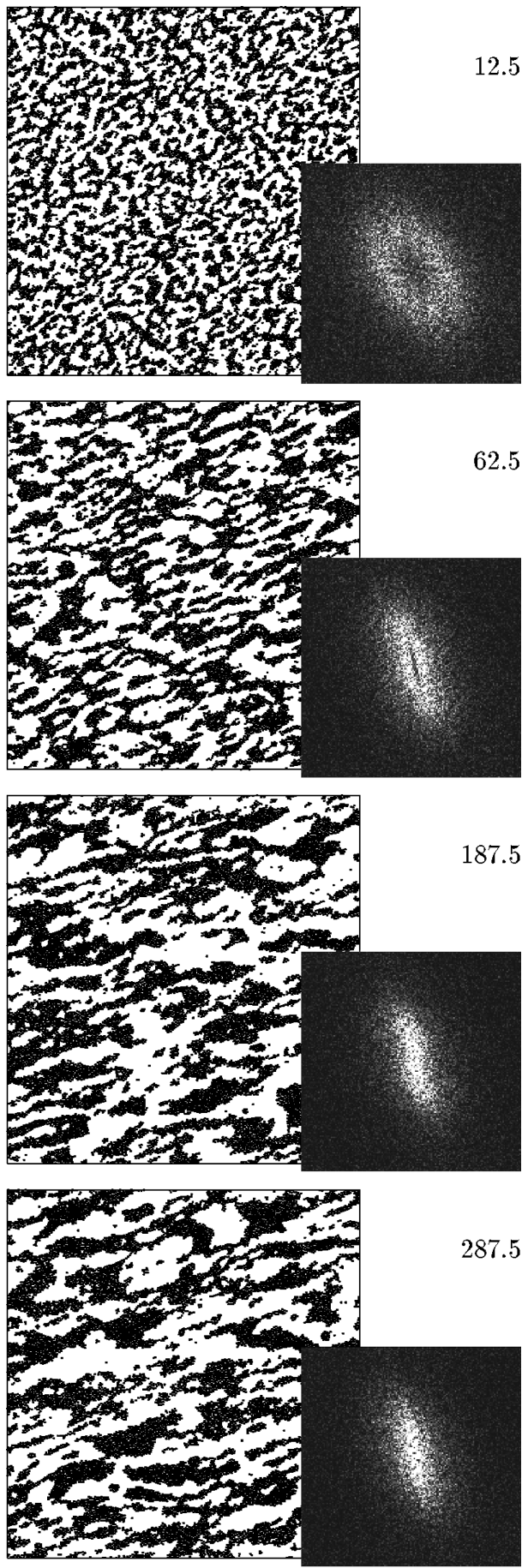

FIG. 3. Time evolution of the domain structure and the scattering patterns at $\dot{\gamma}=0.1$.

$<1$, domain patterns are deformed as if a simple shear deformation is imposed on an isotropic bicontinuous pattern; domains are still randomly fluctuating even in the steady states. When $\dot{\gamma} \tau_{\xi} \simeq 1$, a qualitative change emerges as shown in Figs. 3 or 4 . At even higher shear rates, domains are strongly elongated along the flow direction and exhibit nearly interconnected stringlike structures in the steady states, as seen in Figs. 5 and 6, where $\dot{\gamma} \tau_{\xi}>1$. The corresponding scattering patterns collapse almost to the $y$ axis and
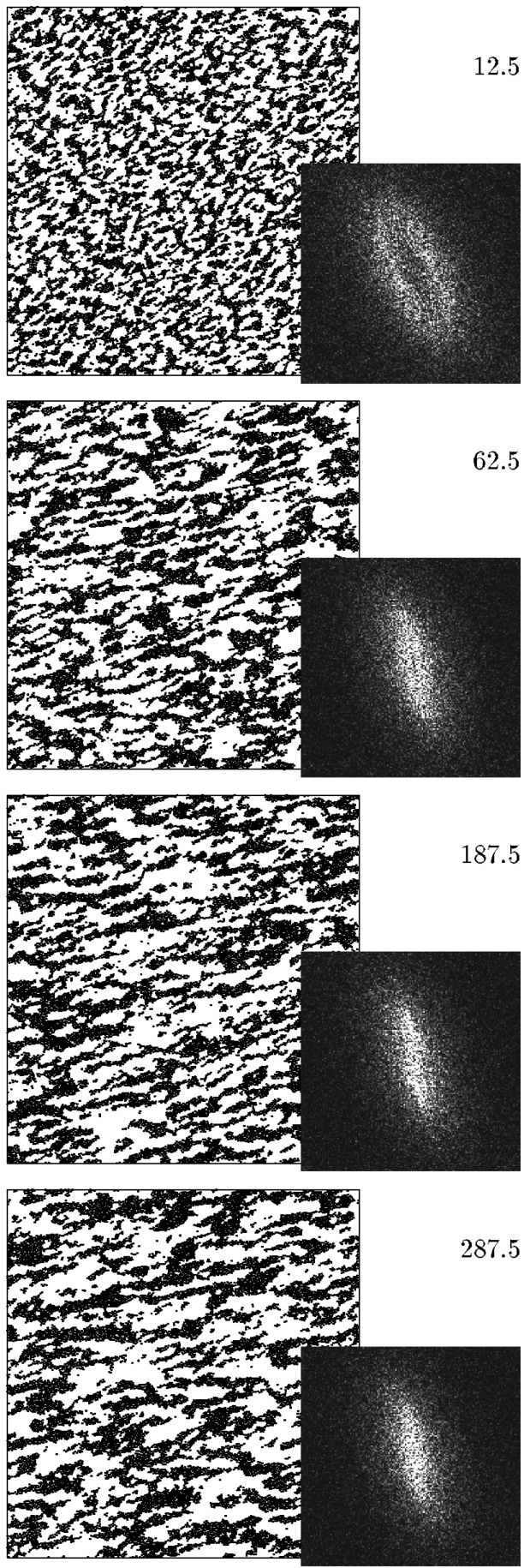

FIG. 4. Time evolution of the domain structure and the scattering patterns at $\dot{\gamma}=0.2$.

show strong streaks in the $y$ direction. Similar domain structures are also observed in polymer mixtures with Reynolds number $\operatorname{Re}<1$ [12], where the structures are called the string phase. To our knowledge, such string phase domain structures have not yet been observed experimentally in the Re $>1$ regime. This simulation gives the first picture of $2 \mathrm{D}$ string phase under shear flow in the $\operatorname{Re}>1$ regime. It is still an open question whether similar 3D domain structures can be observed in real experiments. 

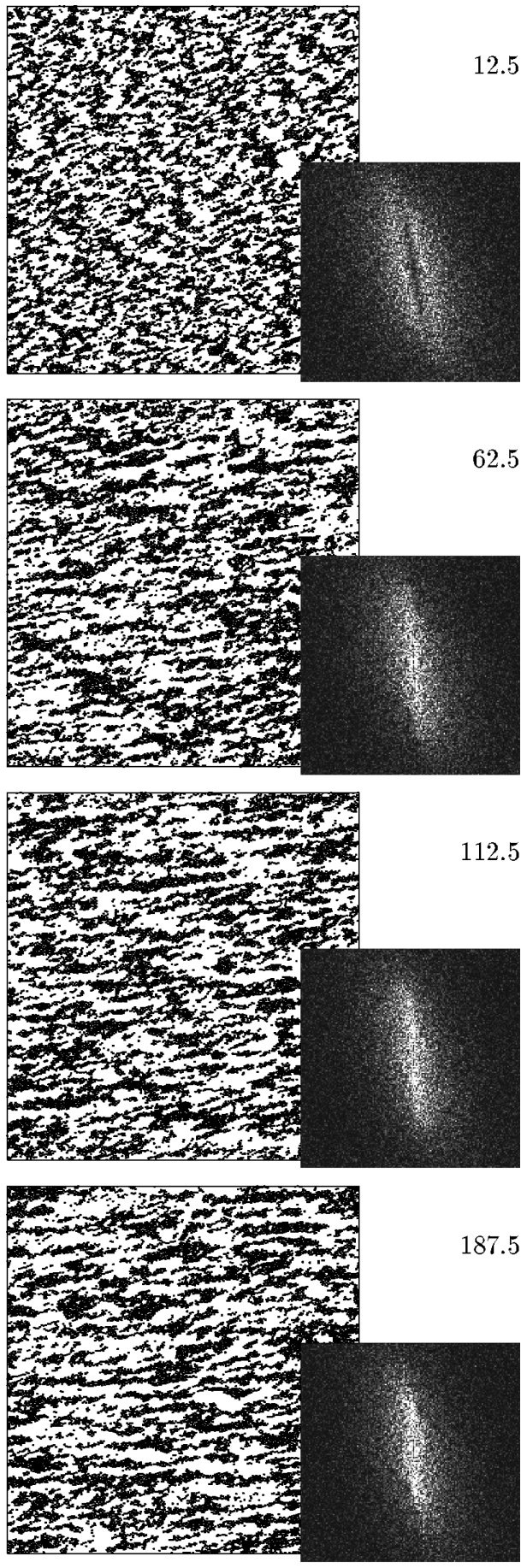

FIG. 5. Time evolution of the domain structure and the scattering patterns at $\dot{\gamma}=0.5$.

\section{B. Rheology}

We also studied rheological properties of the phaseseparating fluid under shear. It is expected that the fluid would exhibit strongly non-Newtonian viscoelastic behavior. The stress tensor is given by

$$
P_{\alpha \beta}=\frac{1}{A}\left[m \sum_{i=1}^{N} v_{i}^{\alpha} v_{i}^{\beta}-\frac{1}{2} \sum_{i}^{N} \sum_{j \neq i}^{N} \frac{r_{i j}^{\alpha} r_{i j}^{\beta}}{r_{i j}} \frac{\partial \phi}{\partial r_{i j}}\right],
$$

where $\alpha, \beta=x, y, A$ is the area of the MD cell, $v_{i}^{\alpha}$ is the $\alpha$ component of the velocity of the $i$ th particle relative to the
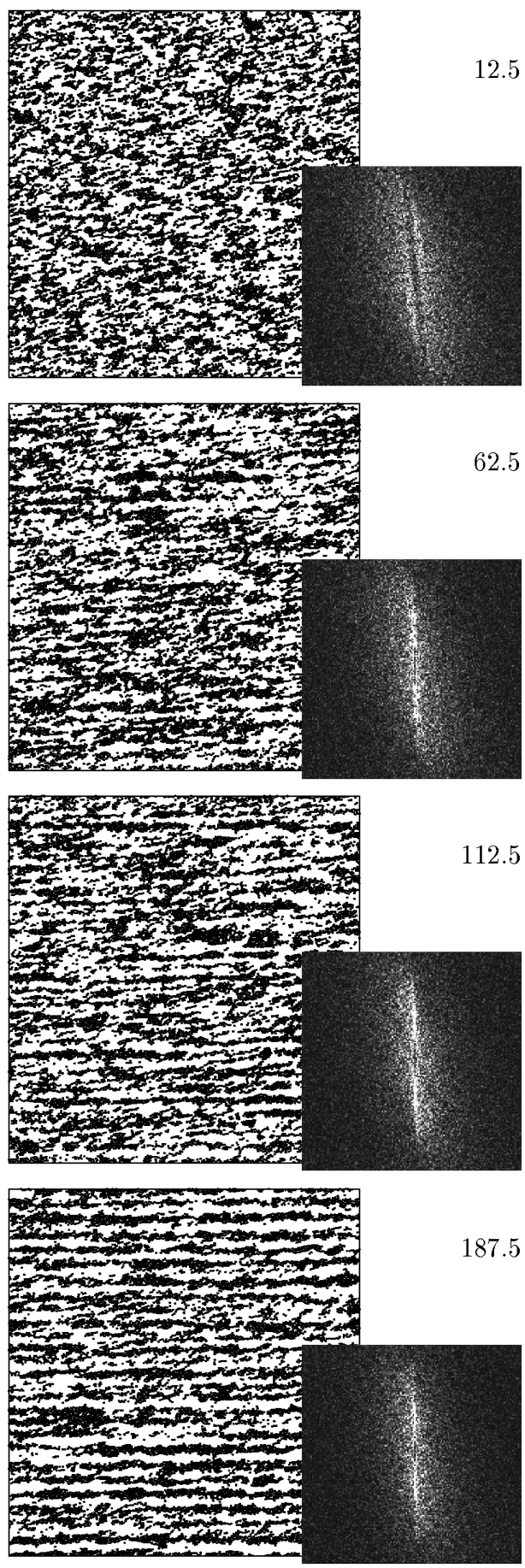

FIG. 6. Time evolution of the domain structure and the scattering patterns at $\dot{\gamma}=1.0$.

average streaming velocity $\dot{\gamma} y_{i} \mathbf{e}_{x}$, and $r_{i j}^{\alpha}$ is the $\alpha$ component of the vector $\mathbf{r}_{i j}=\mathbf{r}_{j}-\mathbf{r}_{i}$. The calculated temporal shear stress $-P_{x y}$ is plotted as a function of the shear strain $\gamma$ $=\dot{\gamma} t$ in Fig. 9 (the quench started at $\gamma=0$ ). The shear stress first increases, showing a peak around $\gamma \simeq 2$, then decreases, and eventually reaches to a stationary value. The initial increase in $-P_{x y}$ is due to domain formation and growth; the subsequent decrease is due to domain elongation along the flow direction. We should point out that large stress fluctuations appearing in the $\dot{\gamma}=0.02$ case may be due to the finite size effect, because the stress increases considerably when 


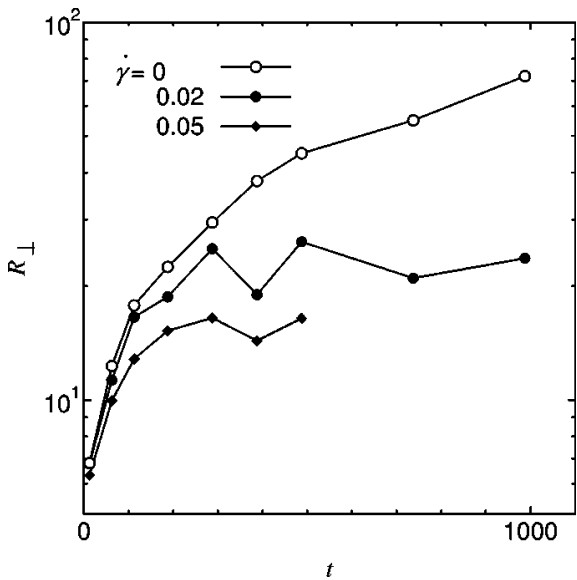

FIG. 7. Time evolutions of $R_{\perp}$ at small $\dot{\gamma}$.

domains are connected through the periodic boundary in the $y$ direction and decreases suddenly when the connections are broken. This behavior may not be seen in the real system. Figure 10 shows the normal stress difference $N_{1} \equiv P_{y y}$ $-P_{x x}$ versus the shear strain $\gamma . N_{1}$ increases first with $\gamma$ because of the anisotropic domain deformation and then levels off to a shear rate-dependent value (in contrast, $N_{1}$ is very small for the one-component fluid). The rheological behavior of the phase-separating fluid mixtures is quite similar to that of viscoelastic fluids such as polymer melts.

The steady state viscosity [25] of the mixture, $\eta_{\text {mix }}$, and that of the one-component fluid, $\eta_{\text {one }}$, are plotted in Fig. 11 as a function of the shear rate. It is easily seen that the fluid mixture shows strong non-Newtonian behavior (i.e., the shear thinning) even at the lowest shear rate, $\dot{\gamma}=0.02$, whereas the one-component fluid shows Newtonian behavior in the region $\dot{\gamma}<0.1$. The excess viscosity from domain contributions can be calculated via $\Delta \eta=\eta_{\text {mix }}-\eta_{\text {one }}$. The shear rate dependence of $\Delta \eta$ is also plotted in Fig. 11. It is found that $\Delta \eta$ increases with decreasing shear rate and can be of the same order as $\eta_{\text {one }}$ for $\dot{\gamma}=0.02$. Our simulation suggests another power-law relation, $\Delta \eta \sim \dot{\gamma}^{-\nu}$ with $\nu \simeq 1 / 2$. In Fig. 12 , we show the shear rate dependence of $N_{1}$. We found that

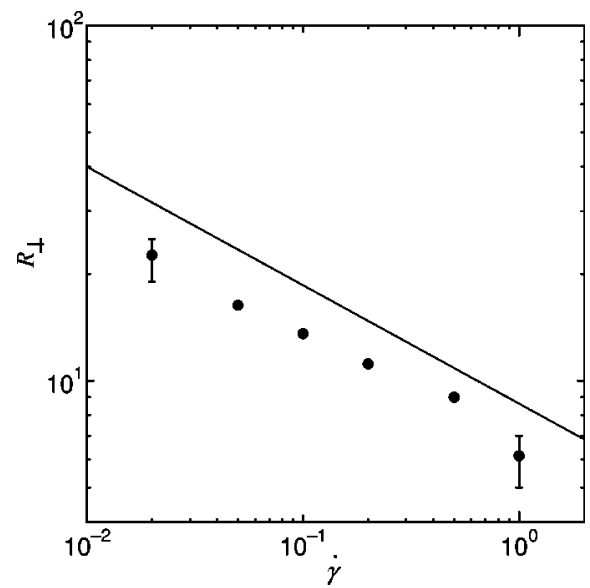

FIG. 8. Shear rate dependence of the shortest average length scale of domain, $R_{\perp}$. The solid line indicates $R_{\perp} \sim \dot{\gamma}^{-\kappa}$ with $\kappa$ $=1 / 3$.

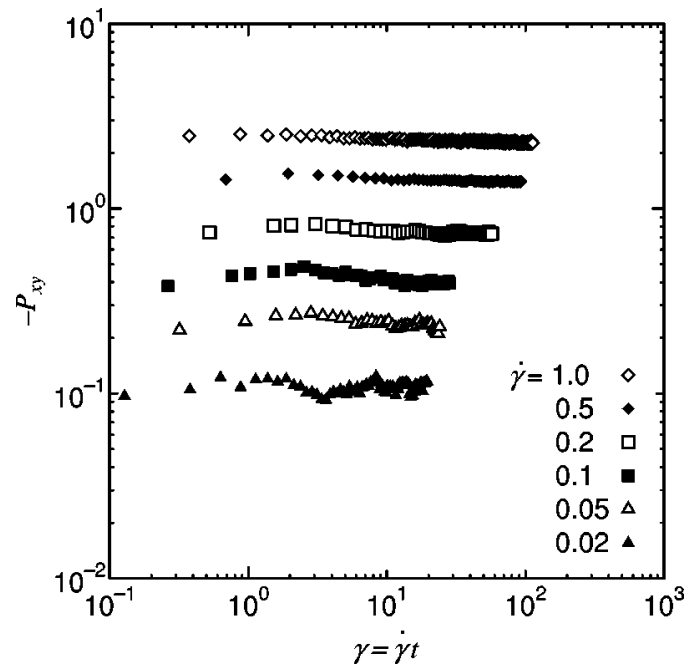

FIG. 9. The plot of the temporal shear stress $-P_{x y}$ versus shear strain $\gamma=t \dot{\gamma}$.

$N_{1}$ increases linearly with increasing shear rate for $\dot{\gamma} \tau_{\xi}<1$; for $\dot{\gamma} \tau_{\xi}>1$, we found $N_{1} \sim \dot{\gamma}^{\mu}$ with $\mu \simeq 1 / 2$.

\section{CONCLUDING REMARKS}

We have performed molecular dynamics simulations in 2D space to study domain structures and rheological properties of phase-separating fluid mixtures under shear flow. In the early stage of the phase separation, we found that anisotropic composition fluctuations emerge and evolve immediately into domains. The higher the shear rate, the stronger the composition fluctuation anisotropy. In the intermediate stage, the anisotropy increases as the domain grows. The phaseseparating fluid mixture eventually reaches a dynamical steady state in which the anisotropic domain structures are preserved. Stringlike domain patterns are seen in the strong shear regime, while randomly fluctuating domain patterns are observed in the weak shear regime. This indicates a possibility of dynamical phase transition occurring at $\dot{\gamma} \tau_{\xi} \simeq 1$. Unfortunately, this tendency has not been detected in $R_{\perp}$.

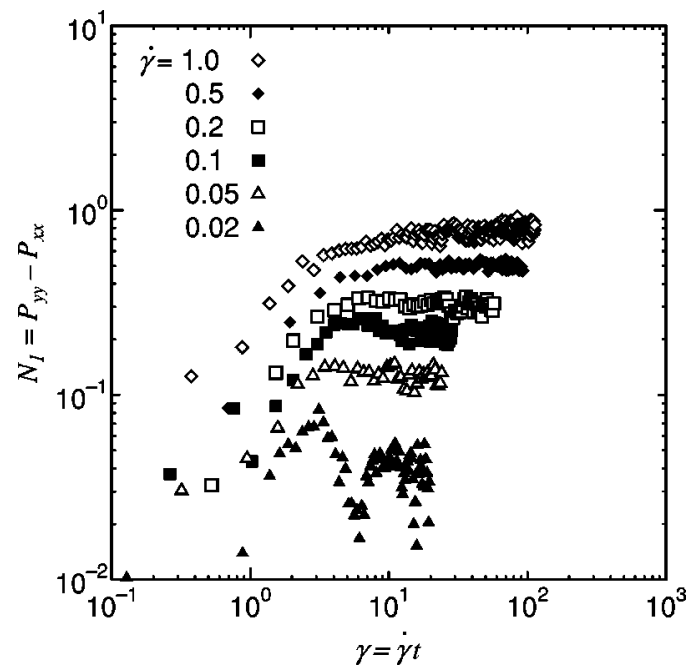

FIG. 10. The plot of the normal stress difference $P_{y y}-P_{x x}$ versus shear strain $\gamma=t \dot{\gamma}$. 


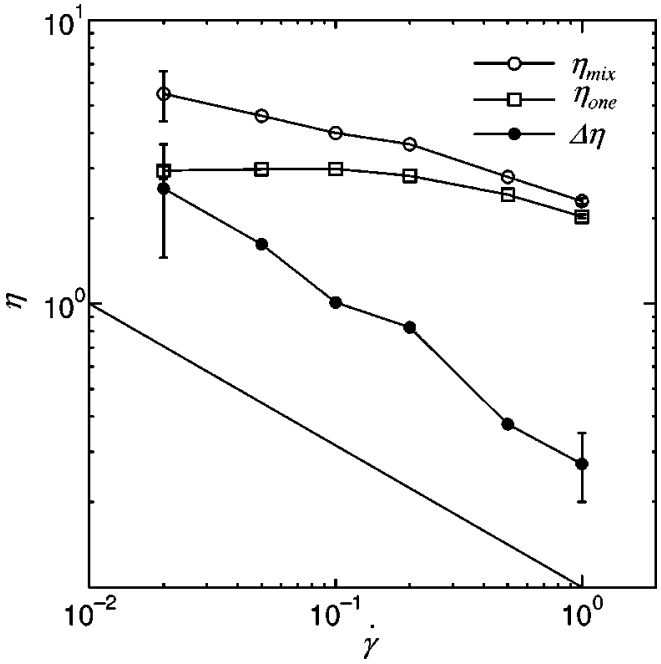

FIG. 11. The steady-state shear viscosity versus the shear rate. Shear thinning is observed for the mixture at $\dot{\gamma}=0.02$. The onecomponent fluid shows purely Newtonian behavior for $\dot{\gamma} \leqslant 0.1$. The excess viscosity $\Delta \eta=\eta_{\text {mix }}-\eta_{\text {one }}$ accounts for most of the shear thinning in $\eta_{\text {mix }}$. The solid line describes a power law $\Delta \eta \sim \dot{\gamma}^{-\nu}$ with $\nu=1 / 2$.

We found that $R_{\perp}$ remains a finite value in the steady state and depends on the shear rate as $R_{\perp} \sim \dot{\gamma}^{-1 / 3}$. The stringlike patterns have not been observed experimentally in the high Reynolds number regime.

We have also studied rheological properties of the fluid mixture. Stress overshoots are observed at $\gamma \simeq 2$, indicating that the decomposing fluid mixture has viscoelastic character. Formation of domains leads to an initial increase in shear stress, but subsequent domain deformation results in a decrease. The fluid mixture also exhibits notable nonNewtonian behavior. The shear thinning is observed even at $\dot{\gamma}=0.02$. In contrast, purely Newtonian behavior is observed for $\dot{\gamma}<0.1$ for the one-component fluid. The excess viscosity

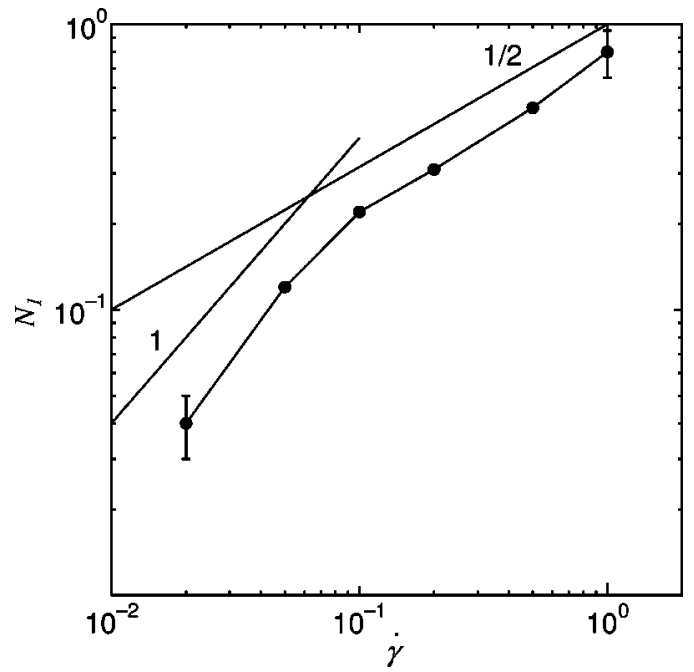

FIG. 12. Shear rate dependence of $N_{1}$. The solid lines describe a power law $N_{1} \sim \dot{\gamma}^{-\mu}$ with two different exponents, $\mu=1$ and $1 / 2$.

$\Delta \eta$ increases with decreasing shear rate and can be of the same order as $\eta_{\text {one }}$ at $\dot{\gamma}=0.02$. Moreover, $\Delta \eta$ can be fitted by a power law, $\Delta \eta \sim \dot{\gamma}^{\nu}$ with the exponent $\nu \simeq 1 / 2$, in both $\dot{\gamma} \tau_{\xi}<1$ and $\dot{\gamma} \tau_{\xi}>1$ regimes. Finally, another power law, $N_{1} \sim \dot{\gamma}^{\mu}$, is also found, where $\mu \simeq 1$ for $\dot{\gamma} \tau_{\xi}<1$ and $\mu$ $\simeq 1 / 2$ for $\dot{\gamma} \tau_{\xi}>1$.

\section{ACKNOWLEDGMENTS}

R.Y. thanks Professor A. Onuki for helpful discussions. This work was supported by a Grant-in-Aid for Scientific Research from the Ministry of Education, Science and Culture. Calculations have been carried out at the Supercomputer Laboratory, Institute for Chemical Research, Kyoto University, and the Computer Center of the Institute for Molecular Science, Okazaki, Japan. X.C.Z. also is thankful for support through NSF and ONR grants.
[1] J.D. Gunton, M. San Miguel, and P. S. Sahni, in Phase Transitions and Critical Phenomena, edited by C. Domb and J. L. Lebowitz (Academic, New York, 1983), Vol. 8.

[2] H. Furukawa, Physica A 204, 237 (1994).

[3] T. Koga and K. Kawasaki, Phys. Rev. A 44, R817 (1991).

[4] F.J. Alexander, S. Chen, and D.W. Grunau, Phys. Rev. B 48, R634 (1993).

[5] W.R. Osborn, E. Orlandini, M.R. Swift, J.M. Yeomans, and J.R. Banavar, Phys. Rev. Lett. 75, 4031 (1995).

[6] S. Bastea and J.L. Lebowitz, Phys. Rev. E 52, 3821 (1995).

[7] W-J. Ma, A. Maritan, J.R. Banavar, and J. Koplik, Phys. Rev. A 45, R5347 (1992)

[8] E. Velasco and S. Toxvaerd, Phys. Rev. Lett. 71, 388 (1993); Phys. Rev. E 54, 605 (1996); M. Laradji, S. Toxvaerd, and O.G. Mouritsen, Phys. Rev. Lett. 77, 2253 (1996).

[9] G. Leptoukh, B. Strickland, and C. Roland, Phys. Rev. Lett. 74, 3636 (1995)

[10] A. Onuki, J. Phys. C 9, 6119 (1997).
[11] C.K. Chan, F. Perrot, and D. Beysens, Phys. Rev. Lett. 61, 412 (1988); F. Perrot, C.K. Chan, and D. Beysens, Europhys. Lett. 9, 65 (1989); C.K. Chan, F. Perrot, and D. Beysens, Phys. Rev. A 43, 1826 (1991); T. Baumberger, F. Perrot, and D. Beysens, Physica A 174, 31 (1991).

[12] T. Hashimoto, K. Matsuzaka, E. Moses, and A. Onuki, Phys. Rev. Lett. 74, 126 (1995).

[13] A.H. Krall, J.V. Sengers, and K. Hamano, Phys. Rev. Lett. 69, 1963 (1992); Phys. Rev. E 48, 357 (1993).

[14] A. Onuki, Phys. Rev. A 34, 3528 (1986); 35, 5149 (1987).

[15] M. Doi and T. Ohta, J. Chem. Phys. 95, 1242 (1991).

[16] T. Ohta, H. Nozaki, and M. Doi, J. Chem. Phys. 93, 2664 (1990).

[17] D.H. Rothman, Phys. Rev. Lett. 65, 3305 (1990); Europhys. Lett. 14, 337 (1991).

[18] C.K. Chan and L. Lin, Europhys. Lett. 11, 13 (1990).

[19] P. Padilla and S. Toxvaerd, J. Chem. Phys. 106, 2342 (1997).

[20] For all physical quantities, the following reduced units are 
used: $\quad R^{*}=R / \sigma, t^{*}=t \sqrt{\epsilon / m \sigma^{2}}, T^{*}=T k_{B} / \epsilon, P^{*}$ $=P \sigma^{2} / \epsilon, \mathbf{v}^{*}=\mathbf{v} \sqrt{m / \epsilon}, \rho^{*}=\rho \sigma^{2} / m, \eta^{*}=\eta \sqrt{\sigma^{2} / m \epsilon}$, and $\dot{\gamma}^{*}$ $=\dot{\gamma} \sqrt{m \sigma^{2} / \epsilon}$. We omit the asterisks in the text and figures to avoid heavy notations.

[21] A.W. Lees and S.F. Edwards, J. Phys. C 5, 1921 (1972).

[22] W.G. Hoover, A.J.C. Ladd, and B. Moran, Phys. Rev. Lett. 48, 1818 (1982); D.J. Evans, J. Chem. Phys. 78, 3297 (1983); D. Brown and J.H.R. Clarke, Mol. Phys. 51, 1243 (1984).

[23] M.P. Allen and D.J. Tildesley, Computer Simulation of Liquids (Clarendon, Oxford, 1987).
[24] D.J. Evans and G.P. Morriss, Phys. Rev. Lett. 56, 2172 (1986).

[25] D.J. Evans and G.P. Morriss, Statistical Mechanics of Nonequilibrium Liquids (Academic Press, London, 1990).

[26] More specifically, we determined $\tau_{\xi}$ by performing a MD simulation at a quiescent state and making the same plot as Fig. 13 of T. Takebe, R. Sawaoka, and T. Hashimoto, J. Chem. Phys. 91, 4369 (1989).

[27] Assuming that the system has only one characteristic length scale $R$ and time scale $\tau$, the replacements $\nabla \rightarrow 1 / R, P$ $\rightarrow \sigma_{s} / R$, and $v \rightarrow R / \tau$ are justified in the Navier-Stokes equation $\nabla P=\eta \nabla^{2} v$. One finally obtains $\tau \sim \eta R / \sigma_{s}$. 